\title{
FREQUENCY STRUCTURE OF RADIO SCINTILLATIONS FOR SEVERAL PULSARS
}

\author{
V.I. KONDRATIEV, M.V. POPOV and V.A. SOGLASNOV \\ Astro Space Center, Profsoyuznaya str., 84/32, Moscow 117997, Russia \\ E-mail:vlad@tanatos.asc.rssi.ru \\ S.V. KOSTYUK \\ Astro Space Center, Profsoyuznaya str, 84/32, Moscow 117997, Russia \\ Moscow State University, Vorobievy Hills, Moscow 119899, Russia
}

\begin{abstract}
Scintillation times and decorrelation bandwidths for the pulsars B0329+54, B1641-45, $\mathrm{B} 1508+55$ and $\mathrm{B} 1919+21$ are determined. The results are based on observations made with different instruments and at different radio frequencies. All objects but the pulsar B $1508+55$ were detected to have more than one frequency scale. The obtained values of scattering parameters are not contrary in general to the Kkolmogorov form of density fluctuation spectrum.
\end{abstract}

\section{Data Processing}

The purpose of the present work is to determine pulsar scattering parameters. We have different sets of observational data (see Table I) for the pulsars B0329+54, B 1641-45, B1508+55 and B1919+21. In all observations undetected signal have been registered with further shifting to zero frequencies and converting to the digital code. Then correction for bandpass amplitude irregularities and dispersion smearing were performed by method of predetection dispersion removal suggested by Hankins in 1971 .

To realize these and subsequent operations some computer software was created which is analogous to the spectrometer with high resolution that allows us to measure very narrow decorrelation bands at low radio frequencies. To analyze the frequency scintillation pattern of investigated pulsars we have calculated crosscorrelation functions (CCFs) between power spectra of the pulses close in time in accordance with the method described in the paper of Popov \& Soglasnov in 1984.

\section{Main Results}

- Scattering parameters for four pulsars were measured (see Table I).

- In general, the parameters obtained are close to the Kholmogorov form of the density fluctuation spectrum. 


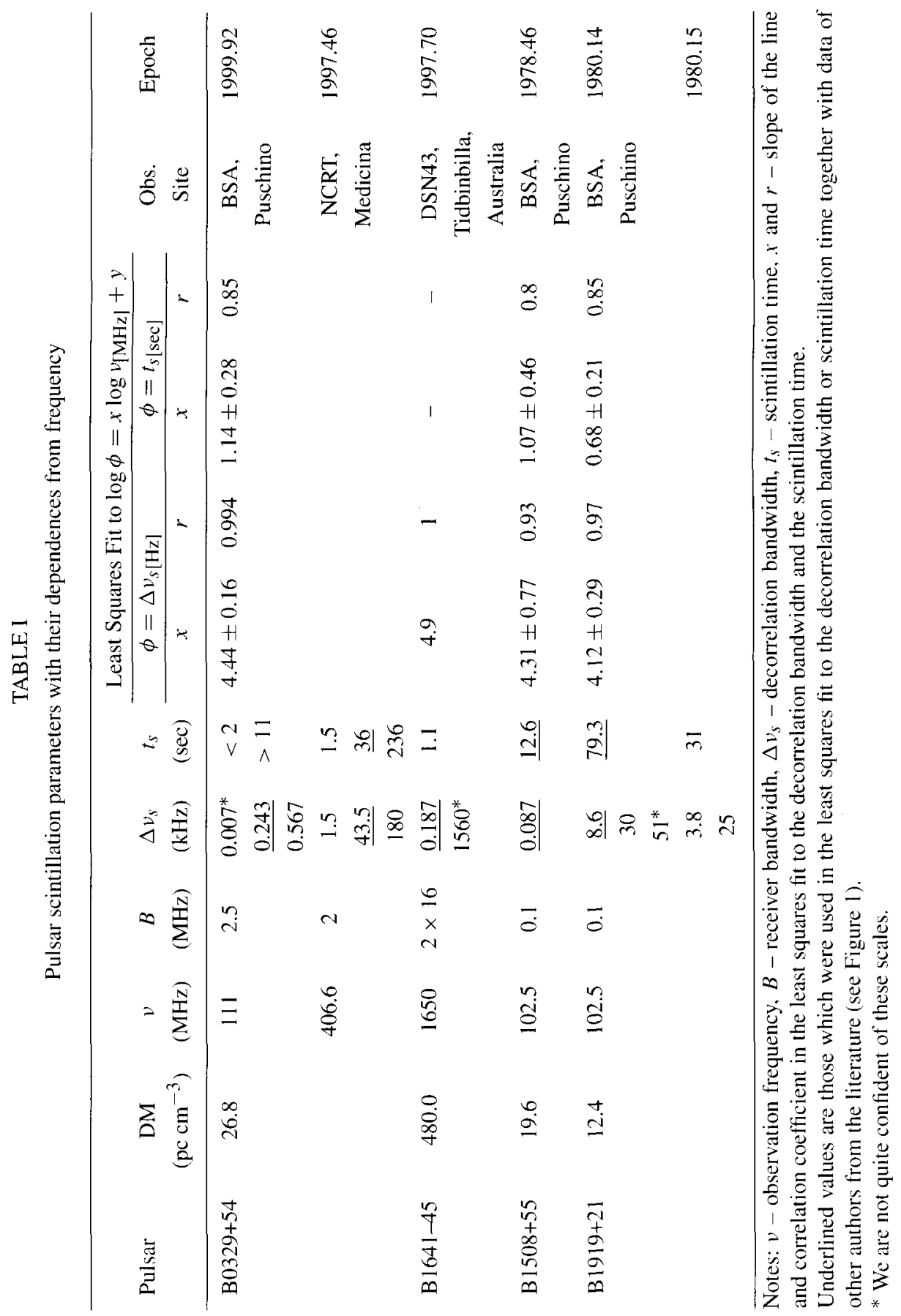




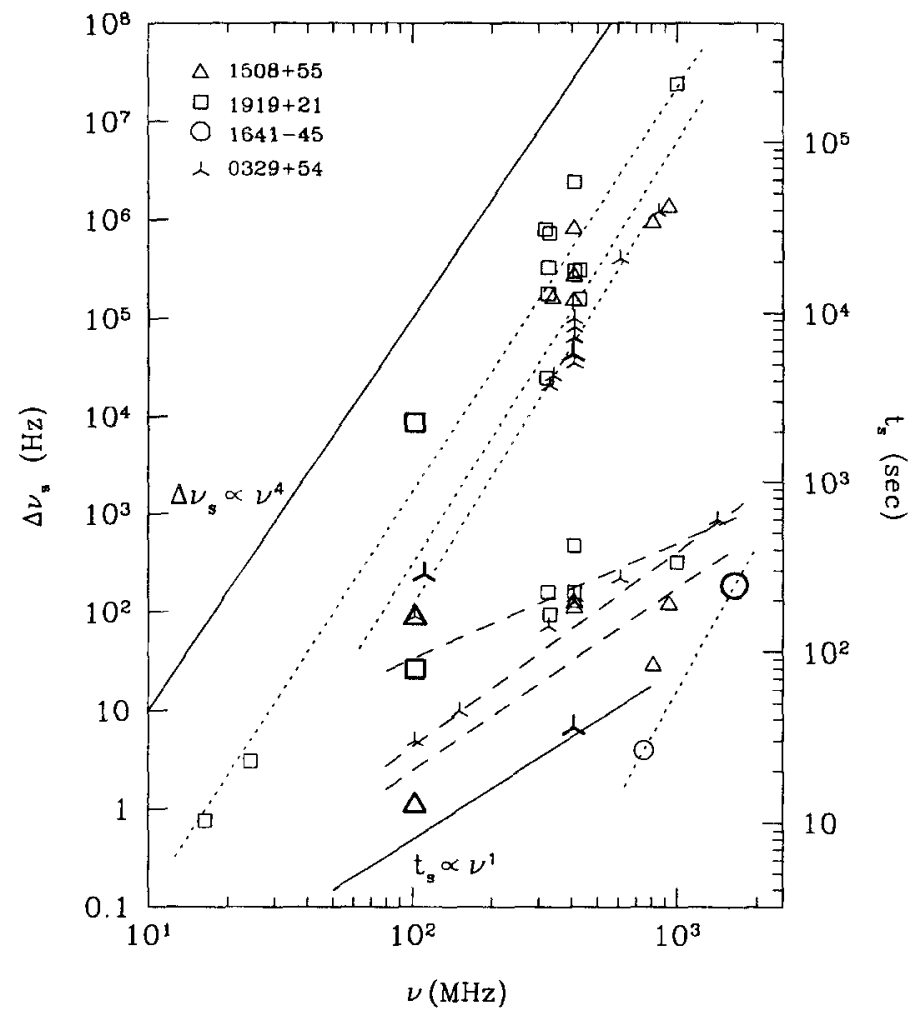

Figure 1. Decorrelation bandwidth $\Delta v_{s}$ and scintillation time $t_{s}$ versus frequency $\nu$ for four pulsars. The data are from the present work (listed in Table I) marked by large bold symbols, and from works of other authors. The dot and long dashed lines are the least squares fits to the decorrelation bandwidth and scintillation time, respectively. The slope of solid lines corresponds to the case of the Gaussian form of the density fluctuation spectrum.

- Large errors in determination of the density fluctuation spectrum form, that corresponds to the large scatter of points in Figure 1, can be due to different frequency scales being measured by different researchers at different radio frequencies.

- Pulsars B0329+54, B 1919+21 and maybe B1641-45 have more than one characteristic frequency scale.

Making some assumptions we could determine the smallest frequency scale for the pulsar $\mathrm{B} 0329+54$ to be $7 \mathrm{~Hz}$, but this value is much smaller than our spectral resolution $(\sim 70 \mathrm{~Hz})$, so we are not quite confident of this value although the expected value of this scale at $406.6 \mathrm{MHz}$ coincides with the measured value at this frequency. The largest frequency scale of the pulsar B1919+21 of $51 \mathrm{kHz}$ seems to be fictitious because there are two scintillation fringes on the dynamic spectrum, which are shifted from each other by just this value. So far as the second scale of the pulsar B 1641-45 is concerned, there are some depressions in the average 
power spectrum which give rise to the second scale in the CCFs. The connection of these depressions with scattering by interstellar plasma is a question because there are many recombination lines of $\mathrm{H}, \mathrm{He}, \mathrm{O}, \mathrm{C}$ at the $158 \alpha, 345 \alpha, 369 \alpha$ and $389 \alpha$ transitions in the frequency range of the observations, which are slightly shifted in frequency, that together with the differential Galaxy rotation could give rise to the line broadening. But it should be noticed that this suggestion needs further analysis.

\section{Acknowledgements}

We are very grateful to Nichi D' Amico (Medicina, Italy), David Del Rizzo (Penticton, Canada), Valery Altunin (Pasadena, USA), Alexander Novikov (Toronto, Canada), Alexey Skulachev (Moscow, Russia), Konstantin Lapaev, Valentina Ivanova, Vladimir Kostromin (Puschino, Russia) and others who have helped us to conduct the observations and to perform the subsequent analysis.

\section{References}

Hankins, T.H.: 1971, Microsecond Intensity Variations in the Radio Emissions from CP 0950, Astrophys. J. 158, 487-494.

Popov, M.V. and Soglasnov, V.A.: 1984, Observations of Interstellar Scattering of Radio Emission of Pulsar PSR 0329+54 at $102.5 \mathrm{MHz}$, Soviet Astron. 28, 424-427. 